\title{
Comparative studies of multiscale edge detection using different edge detectors for MRI thigh
}

\author{
Belinda Chong Chiew Meng, Dayang Suhaida Awang Damit, Nor Salwa Damanhuri \\ Faculty of Electrical Engineering, Universiti Teknologi MARA, Cawangan Pulau Pinang, Malaysia
}

\begin{tabular}{|c|c|}
\hline Article Info & ABSTRACT \\
\hline Article history: & \multirow{11}{*}{$\begin{array}{l}\text { Edge detection plays an important role in computer vision to extract object } \\
\text { boundary. Multiscale edge detection method provides a variety of image } \\
\text { features by different resolution at multiscale of edges. The method extracts } \\
\text { coarse and fine structure edges simultaneously in an image. Due to this, the } \\
\text { multiscale method enables more reliable edges are detected. Most of the } \\
\text { multiscale methods are not translation invariant due to the decimated process. } \\
\text { They mostly depend on the corresponding transform coefficients. These } \\
\text { methods need more computation and a larger storage space. This study } \\
\text { proposes a multiscale method that uses an average filter to smooth image at } \\
\text { three different scales. Three different classical edge detectors namely Prewitt, } \\
\text { Sobel and Laplacian were used to extract the edges from the smooth images. } \\
\text { The edges extracted from the different scales of smooth images were then } \\
\text { combined to form the multiscale edge detection. The performances of the } \\
\text { multiscale images extracted from the three classical edge detectors were then } \\
\text { compared and discussed. }\end{array}$} \\
\hline Received Feb 13, 2020 & \\
\hline Revised Anr 15 2021 & \\
\hline Revised Apr 15, 2021 & \\
\hline Accepted May 30, 2021 & \\
\hline Keywords: & \\
\hline Edge detection & \\
\hline Laplacian & \\
\hline Multiscale & \\
\hline Prewitt & \\
\hline Sobel & \\
\hline
\end{tabular}

This is an open access article under the CC BY-SA license.

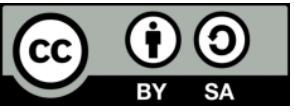

Corresponding Author:

Belinda Chong Chiew Meng

Faculty of Electrical Engineering

Universiti Teknologi MARA, Cawangan Pulau Pinang

Jalan Permatang Pauh, 13500 Permatang Pauh, Malaysia

Email: belinda.chong@uitm.edu.my

\section{INTRODUCTION}

Edge detection is one of the fundamental tools to find the boundaries of an objects for object segmentation and feature extraction [1]. There are two main edge detection operators used for edge detection; the first derivative based and the second derivative based. The first derivative edge detector operator computes the image gradient values to detect image edges. The edges are estimated based on gradient magnitude which is calculated in the $\mathrm{x}$ and $\mathrm{y}$ directions [2]. The second derivative edge detector operator uses zero-crossing for edge detector. The method finds the rate of change in grey intensity and then detects the local maxima in the gradient magnitude. It locates the centres of thick edges and the localization of edges is good [2], [3].

In most cases, the edge pixels are discontinued in the edge detection. This is because the process is based on local change in image intensity. In practical, a connected curve that shows the boundary of an object is desirable for feature extraction. Generally, this type of edge detector is sensitive to noise, and this is because the differential edge detectors behaves as a high-pass filter and thus, it has a tendency to amplify noise [4], [5]. Due to this, it is difficult to differentiate the true and false edges based on the differential edge detector. Multiscale edge detection has attracted a lot of attention in image processing applications due to the fact that edges are multiscale in nature [6]-[8]. The edges extracted from a different scale or resolution are then combined to get the final edge detection result. Multiscale edge detection method has the ability to 
describe the variety of the edge structures [8]-[10]. Wavelet transform is one of the methods often used for multiscale edge extraction. The wavelet coefficients represent the scale of features in an image. It can be used to measure how closely the correlated wavelet is with each section of the image [11]-[13].

S. V Seeri et al. used Haar discrete wavelet transform as multi-resolution method to model the characteristics of textured images [14]. This method well characterized the edges of the textured images. The method fused edges that were extracted from three sub bands which were horizontal $(\mathrm{H})$, vertical $(\mathrm{V})$ and diagonal (D) for edge information. After the edges were extracted, the method also integrated with Sobel edge detector, fuzzy thresholding and morphological operators method to segment and classify the text regions. One of the advantages of the method is that it is able to localize the text regions accurately within the three types of scripts which are English, Kannada and Hindi. Wavelet transform, multiscale method is sensitive to local regularity and it has the limitation of detecting orientation of singularity curve. K. Guo et al. applied framework of Shearlet transform into wavelet transform to improve the edge orientation and curve point detection [15]. The modified method had the ability to estimate the local flatness of an edge and it could also provide detailed information on geometry edge. Obviously, the method has the advantage of nonparabolic scaling and it is able to discriminate features.

Thigh bone is the longest and strongest bone of the entire human body. Various diseases often occur in children and adults. Magnetic resonance imaging (MRI) is an important imaging tool used to investigate the presence of certain abnormalities in thigh [16], [17]. Because of the nature of quantitative MRI imaging data, this requires a considerable amount of human intervention and expertise training opportunities. This process is tedious, time consuming and labour intensive. The development of automatic computer-aided diagnosis system has the additional benefits to help in diagnosing various pathologies [18], [19]. The purpose of this study is to present the formation of multiscale edge detection method that uses three different kernel sizes of averaging filter to smooth image into three different scales. The edges were extracted from three averaging filters that represented coarse to fine scale. Three classical edge extraction operators namely Prewitt, Sobel and Laplacian were used to extract the edges to establish the multiscale edge detection method. The performance of the three classical edge extraction methods was compared.

This paper is organized as follows: in section 2, theories related to averaging filter and edge detection methods on first-order and second-order edge detector operator are presented, while in section 3, an edge extraction method for multiscale edge extraction method is proposed. To clarify, we used three edge extraction operators for the process of multiscale. The experimental results and discussions on the comparison results are then discussed in section 4. Finally, some conclusions are drawn and discussed in section 5 .

\section{AVERAGING FILTER AND EDGE DETECTION METHOD}

Generally, averaging filter is also known as mean filter. It has smoothing effect by average value of the image to reduce noise. On the other hand, classical edge detector is often used for edge detection due to its simplicity [20], [21].

\subsection{Averaging filter}

The implementation of average filtering is to smooth images. The average filter reduces the amount of intensity variation by averaging the pixel itself with the pixel value around its neighbours [22], [23]. Figure 1 shows the kernel averaging operator for $3 \times 3,5 \times 5$ and $7 \times 7$. A $3 \times 3$ square kernel as shown in Figure 1 (a) is often used to smooth images. The smoothing effect produced depends on the size of the averaging filter. Some details in the image will be smoothed by using a $3 \times 3$ averaging filter. The effect of smoothness will be increased by using a 5x5 averaging filter as shown in Figure 1 (b). Next, Figure 1 (c) show the kernel of $7 \times 7$ averaging filter. The smoothing output of a $7 \times 7$ averaging filter will be more than a $5 \times 5$ averaging filter. Therefore, larger kernels can be used for more severe smoothing.

\begin{tabular}{|l|l|l|}
\hline $1 / 9$ & $1 / 9$ & $1 / 9$ \\
\hline $1 / 9$ & $1 / 9$ & $1 / 9$ \\
\hline $1 / 9$ & $1 / 9$ & $1 / 9$ \\
\hline
\end{tabular}

(a)

\begin{tabular}{|l|l|l|l|l|}
\hline $1 / 25$ & $1 / 25$ & $1 / 25$ & $1 / 25$ & $1 / 25$ \\
\hline $1 / 25$ & $1 / 25$ & $1 / 25$ & $1 / 25$ & $1 / 25$ \\
\hline $1 / 25$ & $1 / 25$ & $1 / 25$ & $1 / 25$ & $1 / 25$ \\
\hline $1 / 25$ & $1 / 25$ & $1 / 25$ & $1 / 25$ & $1 / 25$ \\
\hline $1 / 25$ & $1 / 25$ & $1 / 25$ & $1 / 25$ & $1 / 25$ \\
\hline
\end{tabular}

(b)

\begin{tabular}{|l|l|l|l|l|l|l|}
\hline $1 / 49$ & $1 / 49$ & $1 / 49$ & $1 / 49$ & $1 / 49$ & $1 / 49$ & $1 / 49$ \\
\hline $1 / 49$ & $1 / 49$ & $1 / 49$ & $1 / 49$ & $1 / 49$ & $1 / 49$ & $1 / 49$ \\
\hline $1 / 49$ & $1 / 49$ & $1 / 49$ & $1 / 49$ & $1 / 49$ & $1 / 49$ & $1 / 49$ \\
\hline $1 / 49$ & $1 / 49$ & $1 / 49$ & $1 / 49$ & $1 / 49$ & $1 / 49$ & $1 / 49$ \\
\hline $1 / 49$ & $1 / 49$ & $1 / 49$ & $1 / 49$ & $1 / 49$ & $1 / 49$ & $1 / 49$ \\
\hline $1 / 49$ & $1 / 49$ & $1 / 49$ & $1 / 49$ & $1 / 49$ & $1 / 49$ & $1 / 49$ \\
\hline $1 / 49$ & $1 / 49$ & $1 / 49$ & $1 / 49$ & $1 / 49$ & $1 / 49$ & $1 / 49$ \\
\hline
\end{tabular}

(c)

Figure 1. Averaging filter, (a) $3 \times 3$ kernel, (b) $5 \times 5$ kernel, (c) 7x7 kernel 


\subsection{The first order edge detector operator}

Figure 2 shows the Prewitt edge operator model [24], Figure 2 (a) shows vertical direcation, Figure 2 (b) shows horizontal direction. Prewitt edge has one by one model that is used to detect edges in an image. Based on the model, the values that are symmetrical and central (x,y) will make the model take the maximum value that is similar to the detected region.

Figure 3 shows the Sobel edge operator model [25], Figure 3 (a) shows vertical direcation, Figure 3 (b) shows horizontal direction. Sobel operator computes an approximation of edge from the gradient of the image intensity function. Sobel operator gives weight to the point that lies closer to (x, y). As a result, it has smoothing effect and it is also less sensitive to image noise.

\begin{tabular}{|l|l|l|}
\hline-1 & 0 & +1 \\
\hline-1 & 0 & +1 \\
\hline-1 & 0 & +1 \\
\hline
\end{tabular}

(a)

\begin{tabular}{|c|c|c|}
\hline+1 & +1 & +1 \\
\hline 0 & 0 & 0 \\
\hline-1 & -1 & -1 \\
\hline
\end{tabular}

(b)

\begin{tabular}{|l|l|l|}
\hline-1 & 0 & +1 \\
\hline-2 & 0 & +2 \\
\hline-1 & 0 & +1 \\
\hline
\end{tabular}

(a)

\begin{tabular}{|c|c|c|}
\hline-1 & -2 & -1 \\
\hline 0 & 0 & 0 \\
\hline+1 & +2 & +1 \\
\hline
\end{tabular}

(b)
Figure 2. Prewitt edge model, (a) vertical direcation, (b) horizontal direction
Figure 3. Sobel edge model, (a) vertical direcation, (b) horizontal direction

\subsection{The second order edge detector operator}

Laplacian is the second spatial derivative that has a $2 \mathrm{D}$ and isotropic measure of an image. Figure 4 shows the two commonly used Laplacian edge operator models [25], Figure 4 (a) shows outward edges, Figure 4 (b) shows inward edges. The operator enhances the regions of intensity discontinuities and highlights the regions that have rapid intensity change. Thus it is often used to detect fine edges. Due to its characteristic that it is sensitive to noise, the image often is smoothed with Gaussian smoothing filter to reduce noise.

\begin{tabular}{|c|c|c|}
\hline 0 & +1 & 0 \\
\hline+1 & -4 & +1 \\
\hline 0 & +1 & 0 \\
\hline
\end{tabular}

(a)

\begin{tabular}{|c|c|c|}
\hline 0 & -1 & 0 \\
\hline-1 & +4 & -1 \\
\hline 0 & -1 & 0 \\
\hline
\end{tabular}

(b)

Figure 4. Laplacian operator, (a) outward edges, (b) inward edges

\section{THE PROPOSED MULTISCALE METHOD}

The method adapted the concept of multiscale method to extract edges from different resolutions on thigh images. The proposed method is illustrated in Figure 5. Three different kernel sizes of averaging filters were used to smooth the sharpened images and produce three different scales. This was conducted in order to achieve the coarse to fine scale for the multiscale concept. The method involved three main processes which were image sharpening, multiscale and edge extraction by classical edge detectors as shown in Figure 5.

Firstly, the thigh images were sharpened by using Prewitt operator which rotated in eight directions; north, west, south, east, north west, south west, south east and north east. In this process, eight sharpened images were produced. Prewitt operator was chosen due to its its less computation complexity. Prewitt edge detector produces edges which were the most similar to the original images because of its weight on the model.

The concept of multiscale method which included coarse-to-fine edge extraction method based on the size of the averaging filter was then applied to smooth the sharpened images. The eight sharpened images were then smoothed with three different averaging filters which were $3 \times 3,5 \times 5$ and $7 \times 7$. The averaging filter was used to produce images from three different scales which were high scale $(3 \times 3$ kernel), middle scale $(5 \times 5$ kernel) and low scale (7x7 kernel). Edge extraction was then performed on the output of each averaging filter using three different classical edge extraction detectors. The edges extracted from the eight output images from each averaging filter (Prewitt, Sobel and Laplacian) were then combined. A comparison analysis was carried out based on the edge detection from each edge detector. Figure 6 illustrates the steps of the edge extraction and the edges combination for each scale.

The comparison analysis was then carried out for each detector. Figure 6 shows that the output from the three averaging filters were channelled into Prewitt, Sobel and Laplacian edge detectors for edge 
extraction from coarse-to-fine edge extraction. The output of multiscale edge extraction from each edge detector was then compared.

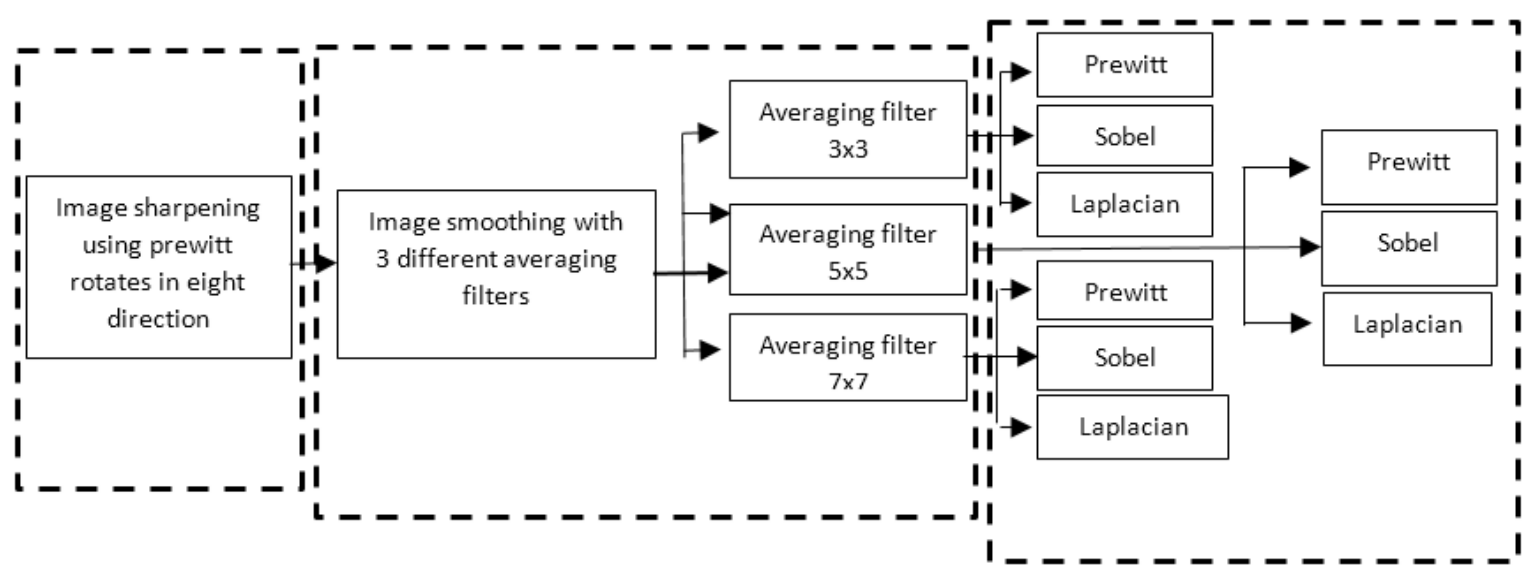

Figure 5. The concepts of multiscale edge extraction method

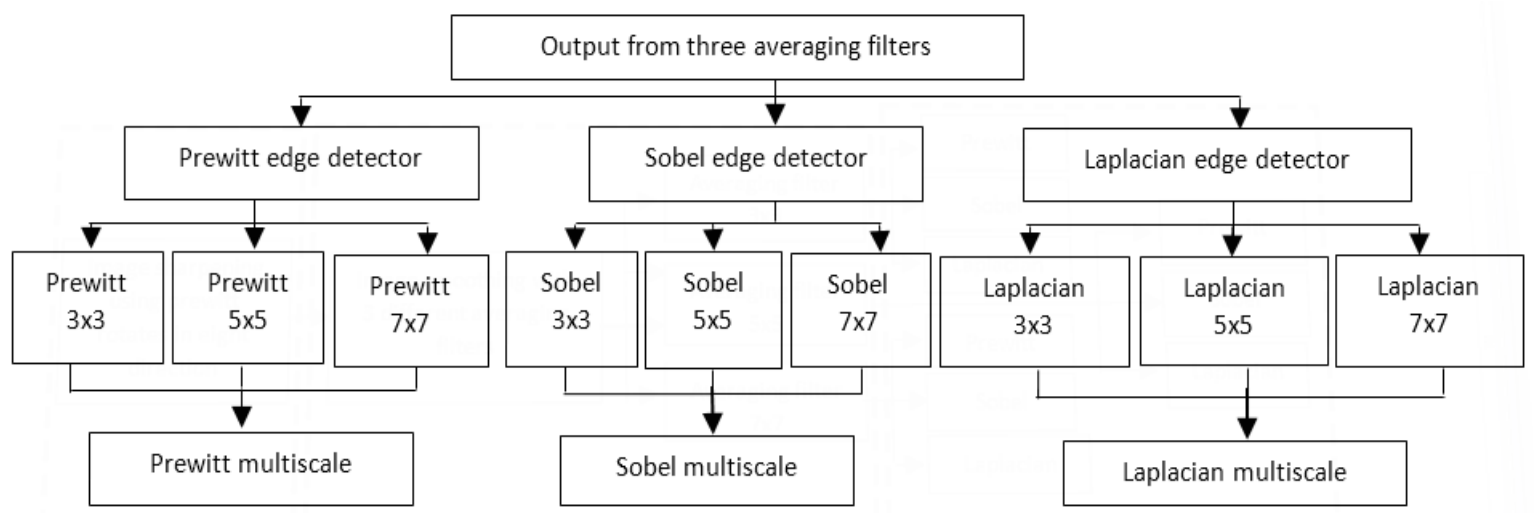

Figure 6. Comparison of multiscale methods

\section{RESULTS AND DISCUSSION}

Edge extraction is a process to detect the boundaries, so that objects can be extracted from the background image. Figure 7 (a) shows the original MRI image. The edges extracted from an edge detector operator often depends on image brightness whereby curved line or edge is extracted when the intensity variation is high in the image. In Figure 7 (b) and 7 (c), it is obvious that the edges extracted from Prewitt and Sobel edge detectors are very similar. The extracted edges from the outlines of the boundaries on muscle, fat and bone morrow are clear. However, it can be seen that some discontinued edges appear in the image. The discontinuity of surface depth and surface orientation cause discontinuity of edges. For Laplacian edge detection, the detector could extract more fine detailed edges as shown in Figure 7 (d). Unfortunately, more discontinued edges were found in the image and the fine detailed edges appear as noise in the image. Generally, the discontinuity of edges could fail to locate important edges that could cause inaccuracy of object extraction.

The multiscale edge extraction method extracted edges over a wide range of scales. When the multiscale edge information fuse together, an edge map could be obtained. The edges information from coarse to fine scale that made the edges were robust. The proposed multiscale edge extraction method is to begin with image sharpening. Images were sharpened using Prewitt compass operator. This was because Prewitt was simple and the edges extracted were very similar to the original image. As shown in Figure 8 (a) to Figure 8 (f), eight images were produced based on eight different orientations. The sharpened images enhanced the edges of the thigh image from eight different orientations. 


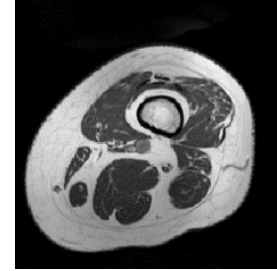

(a)

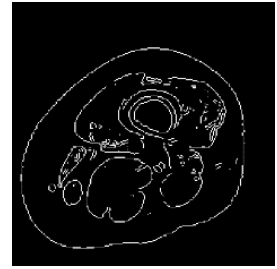

(b)

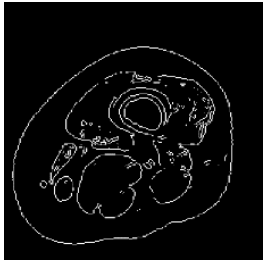

(c)

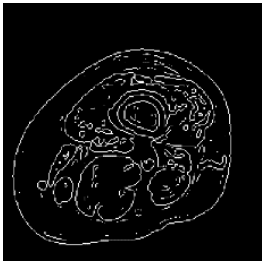

(d)

Figure 7. Edge detection for classical edge detector, (a) original, (b) prewitt extraction, (c) sobel extraction, (d) laplacian extraction

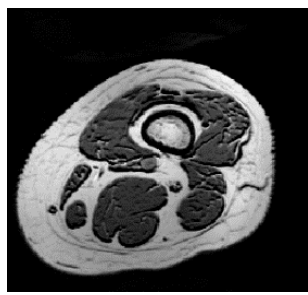

(a)

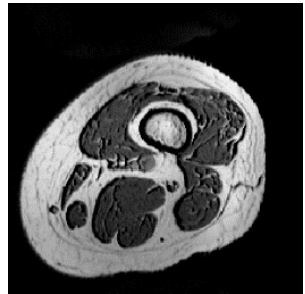

(e)

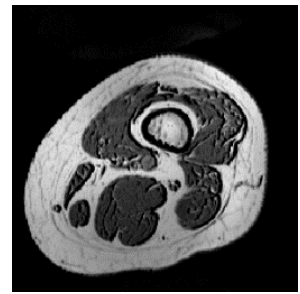

(b)

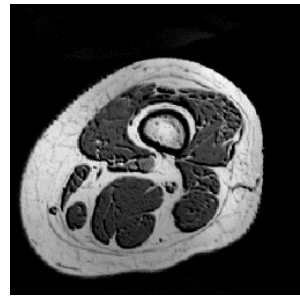

(f)

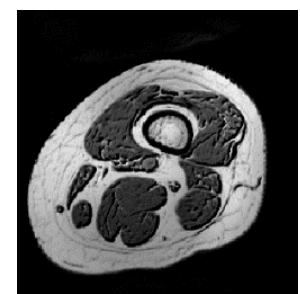

(c)

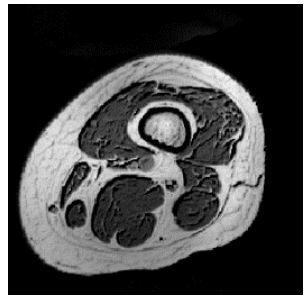

(g)

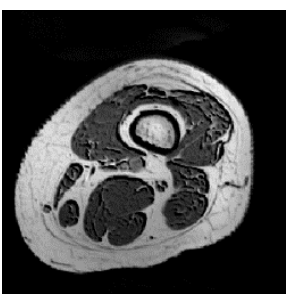

(d)

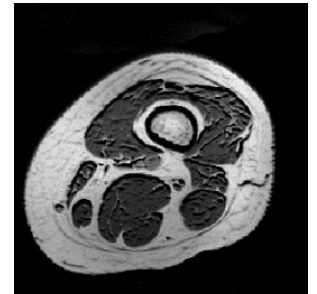

(h)

Figure 8. Images sharpen on eight orientation using Prewitt compass operator, (a) east, (b) north, (c) north east, (d) north west, (e) south, (f) south east, (g) south west, (h) west

Applying the multiscale concept, three differences sizes of average filters were used to smooth the sharpened images for three different scales. Figure 9 (a) shows the original MRI image. Figure 9 (b), Figure 9 (c) and Figure 9 (d) show the output of the averaging filter of 3x3, 5x5 and 7x7 respectively. The output image was a weighted sum of the input pixel. As the mask size increased, the image became blurry and it react to coarse-scale (low scale) methods. The smaller the mask size, it responded more to the spatial accuracy of fine-scale (high scale) methods.

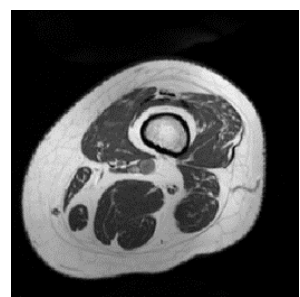

(a)

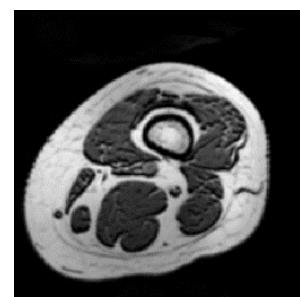

(b)

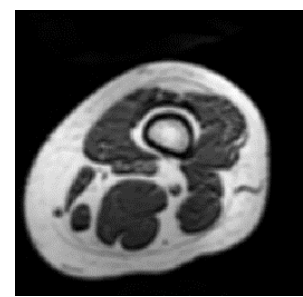

(c)

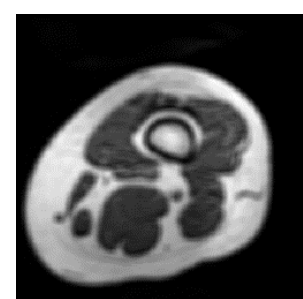

(d)

Figure 9. Three difference of average filters, (a) original, (b) average $3 \times 3$, (c) average 5x5, (d) average $7 \times 7$

Three different classical edge detectors were used to extract the edges from the eight orientations of the sharpened thigh images. The edges extracted from each sharpened orientation image from the same scale were then combined. Figure 10 shows the edge extraction comparison between Prewitt, Sobel and Laplacian edge extraction methods. Figure 10 (a), Figure 10 (b) and Figure 10 (c) show the output of Prewitt 3x3, 5x5 and $7 \times 7$ respectively. Figure 10 (d) is the output of multiscale edge extraction for Prewitt edge detector. Figure 10 (e), Figure 10 (f) and Figure 10 (g) show the output of Sobel 3x3, 5x5 and 7x7 respectively.

Comparative studies of multiscale edge detection using different edge ... (Belinda Chong Chiew Meng) 
Figure $10(\mathrm{~h})$ is the output of multiscale edge extraction for Sobel edge detector. Figure 10 (i), Figure 10 (j) and Figure $10(\mathrm{k})$ show the output of Laplacian 3x3, 5x5 and 7x7 respectively. Figure 10 (1) is the output of multiscale edge extraction for Laplacian edge detector.

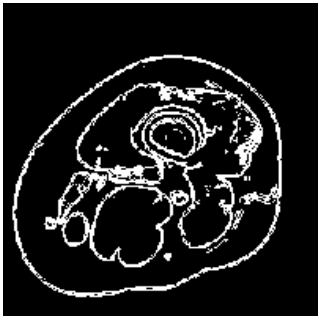

(a)

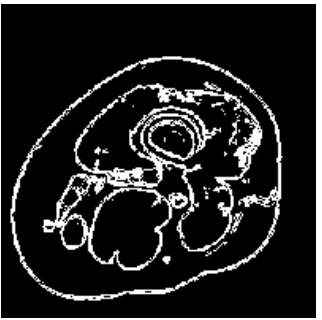

(e)

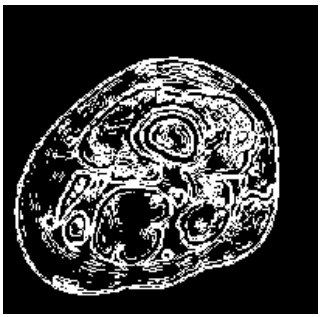

(i)

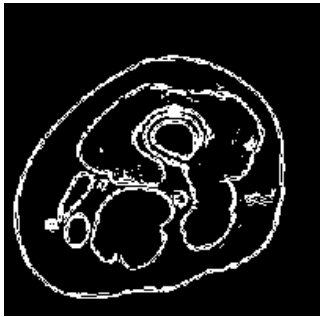

(b)

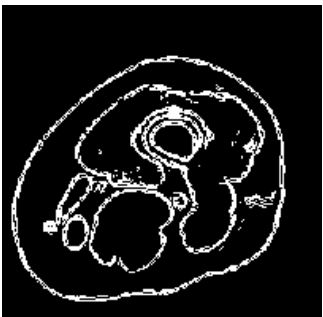

(f)

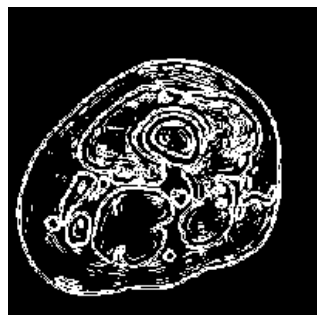

(j)

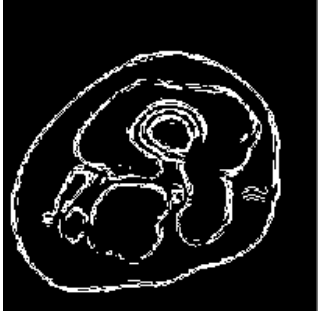

(c)

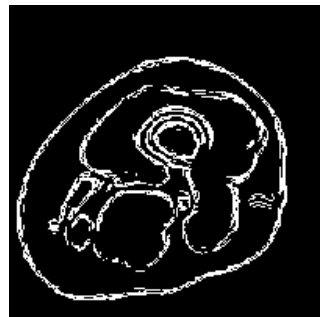

(g)

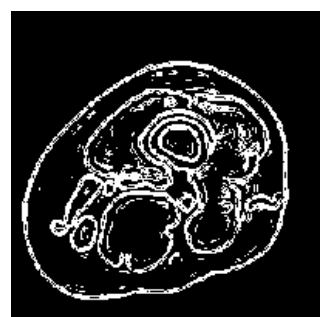

(k)

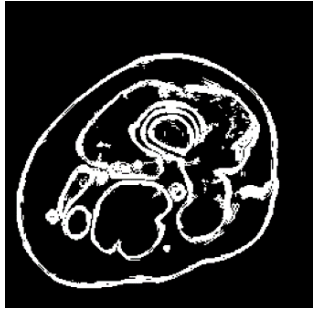

(d)

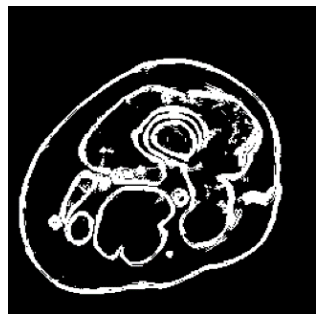

(h)

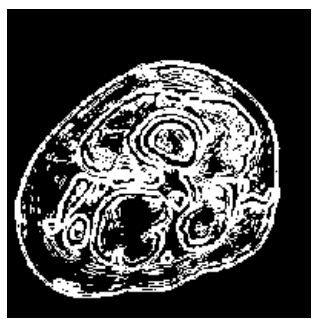

(1)

Figure 10. Result of three multiscale methods, (a) prewitt 3x3, (b) prewitt 5x5, (c) prewitt 7x7, (d) prewitt multiscale, (e) sobel 3x3, (f) sobel 5x5, (g) sobel 7x7, (h) sobel multiscale, (i) laplacian 3x3, (j) laplacian $5 \times 5$, (k) laplacian 7x7, (1) laplacian multiscale

The output of the multiscale edge extraction in Figure 10 (d) for Prewitt detector, Figure 10 (h) for Sobel detector and Figure 10 (l) for Laplacian detector could product continuous edges if compared to Figure 7 (b), Figure 7 (c) and Figure 7 (d). In Figure 10, the resultant multiscale edges can be seen continuous and the boundaries are clearly seen. In the experiment, both Prewitt and Sobel edge detectors produced the edges that were most similar to the original image. Prewitt operator was sensitive to horizontal and vertical edge; Sobel operator was sensitive to diagonal edge. Due to the proposal of multiscale method considering all eight directions for edge extraction, the combination of eight direction edges caused the Prewitt and Sobel operators to produce edges that were similar to the original image.

Laplacian operator is the second derivative operator that the method searches zero crossing to detect edges in an image. As shown in Figure 10, when the kernel size gets bigger, Laplacian 7x7 (Figure 10 (k)) may yield better results in edge localization compared to Prewitt 7x7 (Figure 10 (c)) and Sobel 7x7 (Figure $10(\mathrm{~g})$ ). Besides this, in the comparison analysis, it could be seen that the Laplacian operator was very sensitive to noise as shown in Figure 10 (1). It was due to Laplacian operator attempting to find zero crossing in the process of extracting edges. The fine detailed edges were extracted and amplified that this caused the multiscale edge extracted become noisy by using Laplacian operator. The operator tended to accentuate noise and cause noises to be detected in the background.

Figure 11 shows the comparison of other different parts of thigh. In the figure, it can be seen that the edge extraction from Prewitt and Sobel detectors was close to the original thigh image. However, Laplacian detection contained noise. This can be concluded that Prewitt and Sobel are more suitable for edge extraction on thigh image. The edge extraction preserves the similarity if compared to the original image. 


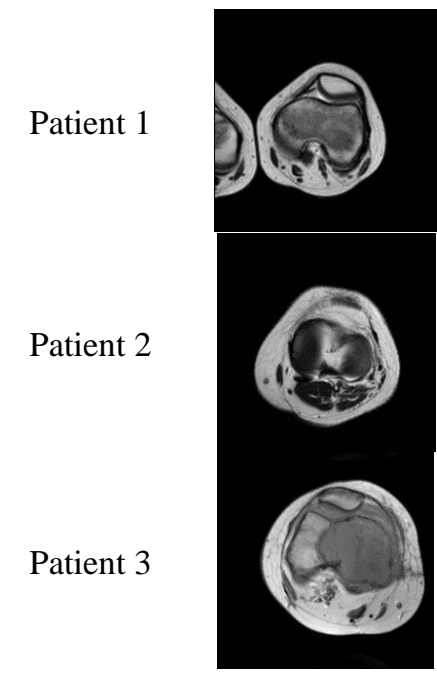

MRI image

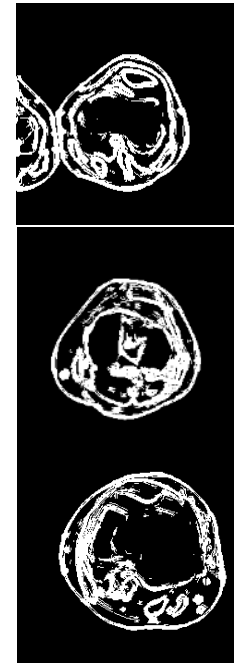

Prewitt

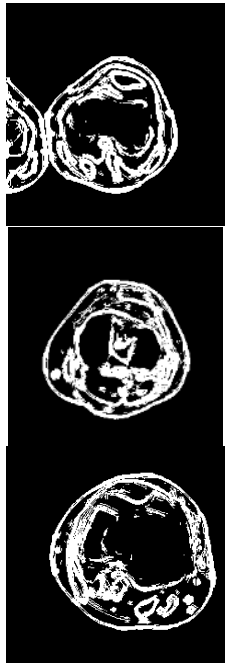

Sobel

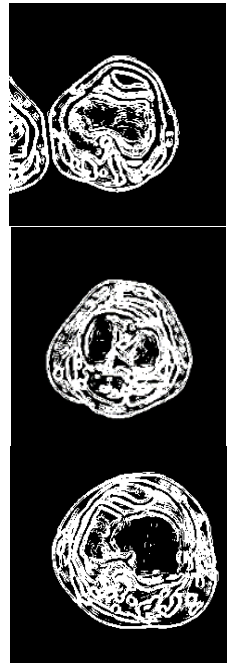

Laplacian

Figure 11. Comparison of multiscale for Prewitt, Sobel and Laplacian on other thigh images

\section{CONCLUSION}

This paper presents a multiscale method that was created by using three different kernel size of averaging filters. The averaging filter smoothed the image and produced three different scales according to the kernel size of the filter. Prewitt, Sobel and Laplacian classical edge detectors were then used to extract the edges at different scales. The performances of these three multiscale edge extractions were then compared. In this study, it can be concluded that Prewitt and Sobel operators could extract edges most similar to the original image. Laplacian could extract fine detailed edges. However, it is sensitive to noise. Laplacian operator could convolve with some smoothing filters to reduce noise in the background. Finally, it can be concluded that the proposed multiscale method that uses either Prewitt or Sobel edge detector as an edge detector offers a great advantage in term of producing multiscale edge detection. In addition, due to its simplicity, the method could reduce computation complexity and more importantly it has good edge preservation and could help in feature extraction. For further improvements, it is recommended that the image should be de-noised before the multiscale process takes place.

\section{ACKNOWLEDGEMENTS}

Authors would like to thank Universiti Teknologi MARA, Cawangan Pulau Pinang for all the research facilities provided for this experiment.

\section{REFERENCES}

[1] D. J. Bora, "A novel approach for color image edge detection using multidirectional Sobel filter on HSV color space,” Int. J. Comput. Sci. Eng, vol. 5, no. 2, pp. 154-159, 2017.

[2] S. Kaur and I. Singh, "Comparison between edge detection techniques," Int. J. Comput. Appl., vol. 145, no. 15, pp. 15-18, 2016, doi: 10.5120/ijca2016910867.

[3] G. Jain and S. Kaur, "A Review on Various Edge Detection Techniques in Distorted Images," Int. J. Comput. Sci. Softw. Eng., vol. 7, no. 5, 2017, doi: 10.23956/ijarcsse/V7I4/0234.

[4] M. Kumar and R. Saxena, "Algorithm and technique on various edge detection: A survey," Signal Image Process., vol. 4, no. 3, p. 65, 2013, doi: 10.5121/sipij.2013.4306.

[5] Y. Aimin, L. Shanshan, L. Honglei, and J. Donghao, "Edge extraction of mineralogical phase based on fractal theory," Chaos, Solitons \& Fractals, vol. 117, pp. 215-221, 2018, doi: 10.1016/j.chaos.2018.09.028.

[6] G. Wang, Z. Wang, and J. Liu, "A new image denoising method based on adaptive multiscale morphological edge detection," Math. Probl. Eng., vol. 2017, pp. 1-11, 2017, doi: https://doi.org/10.1155/2017/4065306.

[7] Y. Du, M. Tong, L. Zhou, and H. Dong, "Edge detection based on Retinex theory and wavelet multiscale product for mine images," Appl. Opt., vol. 55, no. 34, pp. 9625-9637, 2016, doi: 10.1364/AO.55.009625.

[8] B. C. C. Meng, U. K. Ngah, B. E. Khoo, I. L. Shuaib and M. E. Aziz, "A framework of MRI fat suppress imaging fusion system: MRI inhomogeneity correction for femur abnormality analysis," 2015 International Conference on BioSignal Analysis, Processing and Systems (ICBAPS), 2015, pp. 47-51, doi: 10.1109/ICBAPS.2015.7292216.

[9] G. Wang and B. De Baets, "Edge detection based on the fusion of multiscale anisotropic edge strength measurements," in Advances in Fuzzy Logic and Technology 2017, Springer, 2017, pp. 530-536. 
[10] G. Wang, C. Lopez-Molina, and B. De Baets, "Multiscale edge detection using first-order derivative of anisotropic Gaussian kernels," J. Math. Imaging Vis., vol. 61, no. 8, pp. 1096-1111, 2019, doi: 10.1007/s10851-019-00892-1.

[11] O. Prakash, C. M. Park, A. Khare, M. Jeon, and J. Gwak, "Multiscale fusion of multimodal medical images using lifting scheme based biorthogonal wavelet transform," Optik (Stuttg)., vol. 182, pp. 995-1014, 2019, doi: 10.1016/j.ijleo.2018.12.028.

[12] T. Joel and R. Sivakumar, "An extensive review on Despeckling of medical ultrasound images using various transformation techniques," Appl. Acoust., vol. 138, pp. 18-27, 2018, doi: 10.1016/j.apacoust.2018.03.023.

[13] P. S. Gomathi and B. Kalaavathi, "Medical image fusion based on multiscale transforms," J. Med. Imaging Heal. Informatics, vol. 7, no. 2, pp. 478-484, 2017, doi: 10.1166/jmihi.2017.1943.

[14] R. C. Gonzalez and R. E. Woods, "Digital Image Processing," Pearson Education International, 2010.

[15] S. V Seeri, J. D. Pujari, and P. S. Hiremath, "Multilingual text localization in natural scene images using wavelet based edge features and fuzzy classification," Int. J. Emerg. Trends Technol. Comput. Sci., vol. 4, no. 1, pp. $210-$ $218,2015$.

[16] K. Guo and D. Labate, "Microlocal analysis of edge flatness through directional multiscale representations," $A d v$. Comput. Math., vol. 43, no. 2, pp. 295-318, 2017, doi: 10.1007/s10444-016-9486-8.

[17] E. Ahmad, M. Goyal, J. S. McPhee, H. Degens, and M. H. Yap, "Semantic segmentation of human thigh quadriceps muscle in magnetic resonance images," arXiv Prepr. arXiv1801.00415, 2018.

[18] V. Jahmunah et al., "Computer-aided diagnosis of congestive heart failure using ECG signals-A review," Phys. Medica, vol. 62, pp. 95-104, 2019, doi: 10.1016/j.ejmp.2019.05.004.

[19] W. Kovacs, C.-Y. Liu, R. M. Summers, and J. Yao, "Differentiation of fat, muscle, and edema in thigh MRIs using random forest classification," in Proc.SPIE, 2016, vol. 9785, doi: 10.1117/12.2217606.

[20] N. Mathur, S. Mathur, and D. Mathur, "A novel approach to improve sobel edge detector," Procedia Comput. Sci., vol. 93, pp. 431-438, 2016, doi: 10.1016/j.procs.2016.07.230.

[21] J. Bai, C.-C. Chang, T.-S. Nguyen, C. Zhu, and Y. Liu, "A high payload steganographic algorithm based on edge detection,” Displays, vol. 46, pp. 42-51, 2017, doi: 10.1016/j.displa.2016.12.004.

[22] X. Hua, Y. Cheng, Y. Li, Y. Shi, H. Wang, and Y. Qin, "Target detection in sea clutter via weighted averaging filter on the Riemannian manifold," Aerosp. Sci. Technol., vol. 70, pp. 47-54, 2017, doi: 10.1016/j.ast.2017.07.042.

[23] R. R. Datla, "Adaptive fast spatial averaging filter," 2017 8th International Conference on Computing, Communication and Networking Technologies (ICCCNT), 2017, pp. 1-6, doi: 10.1109/ICCCNT.2017.8203929.

[24] N. Imamoglu, J. Gomez-Tames, S. He, D. Gu, K. Kita and W. Yu, "Unsupervised muscle region extraction by fuzzy decision based saliency feature integration on thigh MRI for 3D modeling," 2015 14th IAPR International Conference on Machine Vision Applications (MVA), 2015, pp. 150-153, doi: 10.1109/MVA.2015.7153155.

[25] H. S. Bhadauria, A. Singh, and A. Kumar, "Comparison between various edge detection methods on satellite image," Int. J. Emerg. Technol. Adv. Eng., vol. 3, no. 6, pp. 324-328, 2013.

\section{BIOGRAPHIES OF AUTHORS}

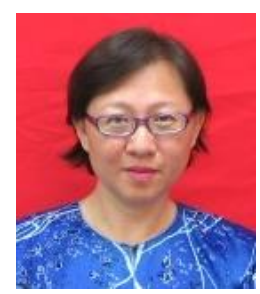

Dr. Belinda Chong Chiew Meng received her B.Sc. (Hons) and M. Eng. in Electrical Engineering in 1997 and 2002 from Universiti Teknologi Malaysia. She received the Ph. D degrees in 2017 from Universiti Sains Malaysia. Currently, she is a senior lecturer at the Faculty of Electrical Engineering, Universiti Teknologi MARA (UiTM), Cawangan Pulau Pinang, Malaysia. Her main research interests include image processing and intelligent system.

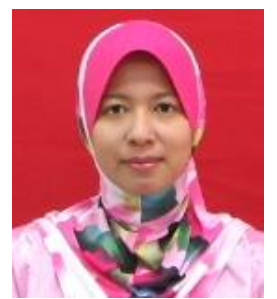

Dayang Suhaida Awang Damit received her B.Eng Hons in Electrical Engineering from Universiti Teknologi Malaysia in 2006 and MSc in Electronic Design from Universiti Sains Malaysia in 2010. She is currently joined the Department of Electrical Engineering (Communication), UiTM as a Lecturer where she occupied herself with teaching and other educational tasks. Her broad research interests are Image Processing, Microwave absorber, and Circuit design.

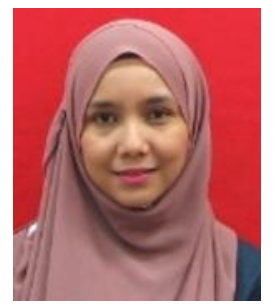

Dr. Nor Salwa Damanhuri received her B.Sc. (Hons) in Electrical \& Electronics Engineering from Universiti Tenaga Nasional (UNITEN) Malaysia. In 2004, she received the Excellence Scheme Programme from MARA to pursue her MSc. in Control Systems Engineering in University of Sheffield, United Kingdom. She had 4 years experiences as a Product Engineer in Freescale Semiconductor Malaysia (fka Motorola Semiconductor) before embark her journey as a lecturer at the Universiti Teknologi MARA (UiTM) Pulau Pinang. She obtained her PhD in Bioengineering from University of Canterbury, New Zealand in 2015. Currently, she is a senior lecturer in Faculty of Electrical Engineering, UiTM Pulau Pinang. Her research interests include lung mechanics, system identification methods, modelling for type 2 diabetic patients and solar PV system. 